CAHIERS DE

NARRATOLOGIE
Cahiers de Narratologie

Analyse et théorie narratives

$16 \mid 2009$

Images et récits

\title{
Littérature et bande dessinée. Enjeux et limites
}

\author{
Jan Baetens
}

\section{OpenEdition}

Journals

Édition électronique

URL : http://journals.openedition.org/narratologie/974

DOI : 10.4000/narratologie.974

ISSN : 1765-307X

Éditeur

LIRCES

\section{Référence électronique}

Jan Baetens, "Littérature et bande dessinée. Enjeux et limites », Cahiers de Narratologie [En ligne], 16 | 2009, mis en ligne le 25 mai 2009, consulté le 30 avril 2019. URL : http://journals.openedition.org/ narratologie/974 ; DOI : 10.4000/narratologie.974

Ce document a été généré automatiquement le 30 avril 2019

\section{(c) (i) (9)}

Cahiers de Narratologie - Analyse et théorie narratives est mis à disposition selon les termes de la licence Creative Commons Attribution - Pas d'Utilisation Commerciale - Pas de Modification 4.0

International. 


\title{
Littérature et bande dessinée. Enjeux et limites
}

\author{
Jan Baetens
}

1 La bande dessinée littéraire est à la mode. Au-delà des collections de bande dessinée consacrée à l'adaptation de textes littéraires, un phénomène qui touche aujourd'hui les éditeurs spécialisés comme les généralistes ${ }^{1}$, au-delà aussi des discussions - terminologiques et autres - lancées par l'importation du label «graphic novel/roman graphique $^{2}$ ", la rencontre des domaines longtemps séparés de la littérature et de la bande dessinée s'est imposée comme une évidence. Non seulement au niveau de la production, où les adaptations sont un exercice populaire depuis plusieurs décennies, mais aussi et surtout au niveau de la manière dont l'organisation du champ littéraire prend depuis peu en compte le décloisonnement de la littérature et de la bande dessinée. Des prix littéraires s'ouvrent au roman graphique (Art Spiegelman et Chris Ware ont ainsi obtenu des récompenses traditionnellement fermées à la littérature visuelle). Des ouvrages de référence ou des aperçus historiques commencent à intégrer la bande dessinée au champ de la littérature (la nouvelle édition du Cambridge History of the American Novel $^{3}$ comportera ainsi, pour la toute première fois, un chapitre autonome sur le "graphic novel ». Les numéros spéciaux de revues littéraires consacrés à la narration graphique sont de plus en plus nombreux, aux États-Unis ${ }^{4}$ comme en Europe ${ }^{5}$. Toutefois, une chose est l'explosion des croisements entre littérature et bande dessinée, autre chose est le bien-fondé ou la solidité de cette nouvelle hybridité, qui ne manque pas de soulever plus d'une question essentielle sur notre conception même du récit.

2 Le succès indéniable des bandes dessinées littéraires ou, plus généralement encore, de la bande dessinée considérée comme une nouvelle forme de littérature, ne devrait pas dissimuler en effet un certain nombre de problèmes fondamentaux, qui représentent les limites actuelles du rapprochement entre les deux médias ou genres.

3 La première difficulté concerne la vision ancienne de l'adaptation, dont la bande dessinée littéraire n'arrive pas toujours à se débarrasser. Comme dans l'approche traditionnelle de l'adaptation, le rapport intermédiatique est réduit à un rapport «binaire » entre une 
œuvre source et une œuvre cible, et de plus ce rapport est toujours pensé comme un rapport «à sens unique »: l'œuvre source est, comme par hasard, toujours un texte littéraire, l'œuvre cible, une bande dessinée. Cette double restriction, que rejettent toutes les théories contemporaines de l'adaptation ${ }^{6}$, survit fortement dans les discours sur la bande dessinée, qui pour la même raison ont aussi beaucoup de mal à se libérer d'une seconde difficulté, à savoir la hantise de la «fidélité », complément inéluctable de toute définition classique de l'adaptation qui prend le texte source non seulement comme modèle mais aussi, implicitement, comme modèle indépassable ${ }^{7}$.

Pour dépasser ce double obstacle (adaptation/fidélité), les études de la bande dessinée littéraire ont sans doute intérêt à s'inspirer de certaines mutations méthodologiques en d'autres domaines, par exemple les études cinématographiques, qui privilégient d'une part une approche résolument culturelle tout en compliquant d'autre part l'approche comparative.

5 S'agissant du premier point, l'adoption du point de vue culturel revient aussi bien à analyser les œuvres adaptées en elles-mêmes (c'est-à-dire hors comparaison directe avec un modèle extérieur) qu'à les étudier comme formes ou pratiques culturelles (c'est-à-dire, dans la tradition désormais bien établie de Raymond Williams, comme des structures en réseau où interviennent un grand nombre de facteurs internes et externes à l'œuvres ${ }^{8}$ ). Dit plus concrètement : dans une telle perspective culturelle, la question n'est plus de savoir si Stéphane Heuet a respecté ou au contraire trahi À la recherche du temps perdu, mais s'il est parvenu à utiliser le langage de la bande dessinée d'une façon intéressante ou non.

En ce qui concerne le second point, le renouveau du regard comparatiste, de nombreux exemples récents en études cinématographiques montrent l'intérêt d'une lecture moins homogénéisante du concept de média. Tout média, qu'il s'agisse du cinéma, mais aussi de la littérature ou de la bande dessinée, devrait apparaître comme une structure hétérogène, à la fois en lui-même et dans ses rapports avec l'environnement médiatique. Comme le démontre clairement la théorie moderne du cinéma ${ }^{9}$, les médias s'avèrent toujours des constructions provisoires et hybrides, dont l'indépendance par rapport à toute une série d'autres médias qui se chevauchent dans des contextes historiques et culturels eux aussi sans cesse changeants, devient vite une vue de l'esprit. La "comparaison" intermédiatique n'est plus dès lors la première des nécessités, contrairement à l'analyse des défis que chaque média pose aux autres médias qui le jouxtent, le concurrencent ou l'étayent à un moment donné. Même le grand débat sur la spécificité médiatique, clé de voûte traditionnelle des analyses intermédiatiques, change ainsi de statut. Sans rien perdre de sa pertinence - car, qu'on le veuille ou non, nous avons toujours besoin de savoir ce que "sont» les médias, même si nous avons abandonné toute ambition essentialiste en la matière -, ce débat se pose maintenant aussi en des termes historiques : toute discussion sur la spécificité d'un média est vu comme le symptôme d'un changement contextuel, qui oblige le média en question à se repositionner dans l'écologie médiatique du moment ${ }^{10}$.

7 On supposera ici que littérature et bande dessinée se rencontrent sur un point très précis : celui du récit. Pareille rencontre est loin d'être la seule possible (il existe par exemple des tentatives de nouer des convergences du côté de la poésie et, de façon sans doute plus stimulante, l'on sait que les bandes dessinées d'avant-garde qui se penchent sur la littérature tentent souvent de freiner le plus possible la dimension narrative des œuvres ${ }^{11}$ ), mais dans le cadre d'une réflexion sur les "limites du récit visuel », cette restriction de champ, provisoire par définition, n'est pas absurde. 
Dès lors, si l'on accepte que la bande dessinée est un récit visuel, il est important de s'interroger sur ce que l'on entend exactement par ce concept dans le contexte du roman graphique ou de la littérature dessinée. Il n'existe pas de réponse simple à cette question, qui suscite en général deux types de réactions, les unes "négatives" (car il est des approches du récit en bande dessinée qu'on refuse catégoriquement), les autres " positives » (et plutôt paradoxales, comme on verra).

Négativement parlant, force est de constater que les discussions sur la bande dessinée comme récit visuel insistent très souvent sur une série de cas de figure que l'on considère comme des exemples ou des pratiques à éviter. Ainsi, on n'apprécie guère les bandes dessinées qui se limitent à «plaquer » des images sur des textes ou qui ne font rien d'autre que de remplir les cases prédécoupées d'une trame narrative déjà entièrement bouclé par un scénario préexistent, conçu et élaboré hors de tout dialogue avec le dessinateur. Certes, ce rejet du scénario « clé sur la porte » et la dégradation artistique du dessin qu'il semble supposer, ne doivent pas être lus comme un plaidoyer en faveur du roman graphique sans scénario, qui s'improvise au fur et à mesure de sa production ${ }^{12}$, mais comme une façon de souligner la plus-value d'une vraie collaboration entre scénariste et dessinateur (idéalement, les deux rôles coïncident dans ce qui s'appelle alors un « auteur complet ${ }^{13}$ »). Un « bon » récit visuel, dans le champ de la bande dessinée, est un récit né de la friction créatrice entre deux médias.

Dans cette rencontre, toutefois, et ceci permet de creuser un peu l'approche "négative " du récit en bande dessinée, il existe une grande réticence à l'égard de certaines formes de correspondance entre texte et image. De même que beaucoup redoutent une soumission trop grande de la bande dessinée au scénario, de même on trouve un refus comparable de tout ce qui, dans l'œuvre achevée, pourrait réduire l'image à un rôle purement illustratif. La forme superlative de cette crainte se manifeste dans le goût de certains, surtout dans les productions d'avant-garde, pour la bande dessinée «muette ${ }^{14}$ ", mais plus généralement la méfiance de toute espèce de domination textuelle est un stéréotype dans la réflexion sur la bande dessinée. D'où, par exemple, le désir de proposer des images «anti-illustratives ", qui s'écartent de ce que décrit le texte (ou, inversement, de rajouter un texte " anti-illustratif » à une œuvre visuelle dont se confirmer ainsi l'indépendance ${ }^{15}$ ). D'où aussi la volonté d'installer une tension entre chaque image et la séquence dont, inévitablement, elle fait partie, comme si l'absorption de la case dans un enchaînement narratif signifiait automatiquement comme une perte de substance (on reviendra sur cette question, qui est capitale pour les dessinateurs, quand bien même leurs avis sont loin d'être convergents sur ce point).

11 Une conclusion provisoire s'impose: dans le contexte des débats sur les narrations graphiques, la manière « négative » d'envisager la question du récit semble indiquer que la qualité d'une bande dessinée ou d'un roman graphique est souvent fonction de son aptitude à rejeter la tutelle du texte (qu'il s'agisse du scénario ou du texte à lire sur la page). Or, comme la question de la qualité ne peut en aucune façon être dissociée de la question sur le statut littéraire ou non du média - car seules les «bonnes » bandes dessinées auront le droit de se faire admettre dans le champ littéraire -, on en arrive à la conclusion plutôt paradoxale que moins une bande dessinée est textuelle, plus elle a de chances d'être perçue comme littéraire.

12 L'analyse de l'approche " positive » confirme ce paradoxe. En effet, par « récit visuel » on entendra ici une bande dessinée capable de développer un récit en exploitant les possibilités narratives de l'image même. Concrètement, ce sont alors trois aspects qui se 
trouvent visés -et qui se renforcent idéalement les uns les autres. Premièrement, l'image est censée être construite de telle façon qu'en dépit de son caractère fixe elle génère une lecture de type narratif, qu'elle représente ou non un « instant décisif ». Deuxièmement, l'effet narratif doit découler aussi de la mise en séquence des images, qui à elle seule, par ses effets de montage, contribue à un renforcement du récit (à première vue, ce point paraît se négocier difficilement avec le précédent, mais l'approche "négative » du récit en bande dessinée a permis de signaler que les «bons » romans graphiques arrivent à maintenir à la fois la focalisation sur la case et celle sur la séquence). Troisièmement, une valeur spéciale est également accordée aux romans graphiques qui, tout en étant publiés comme livres, parviennent à sauvegarder quelque chose de la dynamique temporelle qui caractérisait le genre au moment où sa forme de publication dominante était encore le feuilleton, prépublié dans les journaux ou en magazine ${ }^{16}$.

Si l'on voit assez quel peut être l'apport de ces trois dimensions à la mise en place d'une temporalité propre à la bande dessinée et partant quelle est l'importance de ces éléments dans les débats sur le caractère littéraire - c'est-à-dire, répétons-le, qualitatif du roman graphique, le renvoi à la littérature servant ici comme un label de qualité et vice versa -, on se rend compte tout aussi facilement des dangers que soulève chacune d'elles. D'abord, mettre l'accent sur le potentiel narratif de l'image peut conduire à ce que d'aucuns appellent l'esthétique du poster; favoriser la belle image isolée serait oublier les contraintes du média, qui impliquent nécessairement la série et la séquence. Ensuite, aligner les effets de transition entre cases sur les lois du montage au cinéma amène pareillement à perdre de vue la spécificité du média, qui se tromperait de cible à vouloir copier coûte que coûte les moyens de l'image mobile -comme si l'image mobile était en soi plus performante que l'image fixe en termes de narrativité ${ }^{17}$. Enfin le retour à la poétique du feuilleton, pour peu qu'on le fasse jouer contre la logique du livre, risque toujours de minimiser le jeu sur les structures d'ensemble et le surplus apporté par la possibilité de construire un récit qui dépasse l'élaboration au jour le jour.

S'il fallait résumer cette analyse, on pourrait dire que la bande dessinée littéraire se pense essentiellement en relation avec un certain type de récit, plutôt qu'en relation avec des critères plus formels comme le format "livre» (plutôt que le format "album»), le nombre de pages (n'importe quel nombre de pages, pourvu qu'il échappe au standard commercial des 48 ou des 62 pages), le fait qu'il constitue une publication indépendante (plutôt que de faire partie d'une série, comme la bande dessinée traditionnelle) ou encore du contenu spécifique (la thématique " adulte », souvent d'inspiration autobiographique, n'est pas une fatalité). Toutefois, ce récit est défini en partie contre le texte et, peut-être, la littérature au sens classique du terme : si le récit d'une bande dessinée ou d'un roman graphique n'est pas d'abord visuel, selon les modalités qu'on vient de passer rapidement en revue, les chances qu'il soit qualifié de littéraire, semblent diminuer considérablement.

Il ne peut toutefois suffire d'étudier la bande dessinée littéraire en elle-même. L'enjeu fondamental des questions soulevées par l'intérêt contemporain pour la rencontre de deux médias devrait être la remise en question de nos quasi-certitudes sur ce que sont la bande dessinée d'une part et la littérature d'autre part.

Le roman graphique, que beaucoup considèrent comme un genre nouveau mais déjà solidement établi, doit tout d'abord nous forcer à repenser nos idées sur la bande dessinée en général, dont les aspects narratifs ont peut-être été relégués au second plan depuis la percée des études sémiotiques et formalistes. Certes, le succès du roman 
graphique n'est pas le seul élément qui occasionne ce retour aux racines narratives du média. La bande dessinée littéraire participe d'un regain d'intérêt plus général pour la chose narrative (renouveau de la narratologie, explosion postmoderne des microrécits, exploitation du storytelling dans le domaine du marketing, domination sans cesse plus massive des médias visuels qui n'ont pas connu le « soupçon » du récit caractéristique de la littérature de recherche, et ainsi de suite), mais elle peut à coup sûr apporter quelque chose de très spécifique, à savoir l'attention particulière donnée à l'articulation nécessaire -en même temps qu'inévitable- entre structure narrative et structure médiatique - ce que Philippe Marion appelle la «médiagénie» du discours narratif ${ }^{18}$. Le retour du récit n'est pas le retour à un récit indifférencié, insensible aux frontières, si floues et changeantes soient-elles, entre les médias. En ce sens l'exemple du roman graphique, où l'on voit très facilement comment le choix d'un média façonne le récit (surtout quand il s'agit de romans graphiques qui adaptent et modifient un matériau littéraire déjà existant), peut aider à reposer sur de nouvelles bases les vieilles questions de la spécificité de la parole littéraire.

En second lieu, la bande dessinée littéraire peut également nous inciter à réexaminer d'un œil critique certains concepts de base des études littéraires. On voudrait pour terminer donner deux exemples d'une telle relecture: d'abord la question du narrateur autobiographique, puis celle des techniques de composition du feuilleton.

Dans le domaine littéraire proprement dit, les recherches sur l'autobiographie continuent à favoriser la réflexion sur le "pacte autobiographique ", c'est-à-dire l'identité supposée des positions de l'auteur, du narrateur et du personnage. La plupart des analyses menées dans cette perspective analysent les figures de discours - production et réception confondues - permises par le «jeu» entre ces trois instances, et il n'est bien entendu nullement question de critiquer ni la pertinence, ni le grand intérêt de ce point de vue. Toutefois, le roman graphique permet de renouveler ce débat d'une manière à la fois profonde et naturelle. Profonde, parce que la bande dessinée littéraire va introduire de nouvelles distinctions qui vont toutes dans le sens d'une plus grande polyphonie de la parole narrative. Naturelle, parce qu'elle n'a pas besoin pour cela de pratiquer ou d'inventer des formes qui s'écartent de la norme autobiographique, comme l'autofiction en littérature, par exemple. Il suffit au roman graphique de suivre sa propre médiagénie pour qu'émergent tout de suite des variantes tout à fait passionnantes du pacte autobiographique.

19 À cet égard, un rôle clé est joué par le dédoublement de l'énonciation, qui se divise en deux versants : d'une part l'énonciation verbale ou « narration » (toujours présente, sauf dans le cas des bandes dessinées muettes - exemple à mon sens non (encore ?) attesté dans le domaine du roman graphique autobiographique); d'autre part l'énonciation visuelle, que Philippe Marion nomme " graphiation ${ }^{19}$ » et qui fait partie intégrante de la médiagénie du genre (encore qu'il soit toujours pensable - mais pas pour autant très enrichissant - d'imaginer un roman graphique n'ayant que des cases vides légendées). Or, ce qui constitue l'intérêt de cette division, c'est que, contrairement à ce qui se passe pour les trois instances (auteur, narrateur, personnage) du pacte autobiographique, il n'est ici pas possible de fixer en termes d'identité ou de non-identité les rapports entre narration et graphiation. Certes, on peut toujours essayer de dessiner comme on écrit, ou penser qu'on le fait, mais au niveau de la lecture il existe souvent un sentiment de décalage qui n'a rien à voir avec des questions d'état civil : même dans les cas où celui qui dessine et celui qui écrit sont la même personne, en l'occurrence celle de l'auteur, il n'est pas sûr 
que le lecteur perçoive de la même façon les informations fournies par la narration et celles fournies par la graphiation, si bien que l'instance narrative d'un roman graphique sera (presque) toujours lue de manière plus «polyphonique » que l'instance narrative d'un texte littéraire non visuel. Certains lecteurs pourraient utiliser une telle observation pour revenir sur la position du narrateur littéraire, pour s'interroger sur de possibles effets polyphoniques obnubilés par l'accent trop exclusif mis sur la question de l'identité entre narrateur, auteur et personnage.

Ce qui rend le cas de l'autobiographie dessinée également très stimulant, c'est que de nombreux exemples complexifient encore davantage - mais une fois de plus de manière tout à fait «naturelle »- le possible clivage entre narration et graphiation. C'est ce qui arrive lorsque la main du dessinateur et la voix du personnage n'appartiennent pas à la même personne, chose moins rare qu'on ne le pense dans l'autobiographie dessinée. Un bel exemple est la trilogie La guerre d'Alan d'Emmanuel Guibert ${ }^{20}$, où l'auteur dessine la vie d'un GI américain, Alan Ingram Cope, et que cette vie est non pas une biographie mais une autobiographie - par dessinateur interposé - puisque le récit est fait par le personnage authentique qui s'exprime à la première personne. Ici encore, l'acte narratif du roman graphique se transforme en un palais aux miroirs qui accroît encore l'effet polyphonique inhérent à la duplicité entre narration et graphiation (dans le cas de Guibert, ce clivage est une pièce essentielle de la machine rhétorique du texte, puisque le « dit » et le "montré », sans diverger radicalement, sont systématiquement décrochés l'un par rapport à l'autre, à la fois sur le plan temporel - le rythme de la narration et celui de la graphiation n'est pas le même - et sur le plan stylistique - le ton des deux est également sujet à de subtiles différences).

Des remarques similaires peuvent se faire au sujet du deuxième et dernier exemple, qui concerne la technique du feuilleton. Ici encore, les pratiques tout à fait courantes du roman graphique peuvent aider à renouveler notre vision du feuilleton, qui est devenu aujourd'hui soit un divertissement littéraire, soit un anachronisme compromis par les mauvais souvenirs du mélodrame du XIXe siècle. La littérature dessinée demeure en effet en grande partie une narration caractérisée par les contraintes du feuilleton (la prépublication en chapitres ou fragments est tout sauf rare, même lorsque le roman graphique est pensé prioritairement en fonction du format « livre») et il y a tout lieu de croire que certaines pratiques bien rodées de la bande dessinée peuvent jeter une nouvelle lumière sur nos approches du feuilleton.

Inévitablement, le grand modèle est ici Hergé (qu'on ne s'étonnera plus, il faut l'espérer, de voir rangé parmi les tenants de la bande dessinée « littéraire »). Grand feuilletoniste, Hergé apporte à l'étude du feuilleton au moins trois grandes leçons. Pour commencer, il est un de ceux qui ont redéfini de fond en comble les lieux stratégiques de la poétique de la surprise ${ }^{21}$ en feuilleton. Au lieu de se concentrer sur la seule dynamique de la chute surprenante suivie d'une relance à la fin, puis au début de chaque nouvelle unité, Hergé a mis en place un système qui met quelque peu en sourdine l'effet d'attente en bas de chaque planche et l'effet de résumé ou de relance en haut de chaque planche pour accentuer en revanche les possibilités de surprise narrative à la fin de chaque strip (et comme une planche habituelle des Aventures de Tintin compte quatre strips, on trouve donc quatre variations sur le principe du cliffhanger, puis de la relance au début du strip suivant). Les lieux stratégiques de la surprise narrative sont ainsi à la fois multipliés (l'effet de surprise se diffuse, il ne reste pas l'apanage des seules frontières entre unités) et diminués (en importance, car si la recherche de la surprise se généralise, les attentes et 
surprises en question doivent se faire plus discrètes, sous peine de rendre le récit peu crédible : un deus ex machina à chaque strip déboucherait vite sur le grand guignol ${ }^{22}$ ).

À cela s'ajoute qu'Hergé est également celui qui a travaillé plus que d'autres l'importance de la zone de "tension basse» entre les lieux stratégiques que sont, en régime de feuilleton, l'effet d'annonce et l'effet de surprise. Comme le champ d'action de sa poétique de la surprise est le strip, et non plus la planche, la zone tampon entre annonce et surprise est extrêmement réduite. Dans bien des cas, il ne reste en effet qu'une seule case entre celle de gauche (où se révèle l'information surprenante) et celle de droite (où s'installe l'effet d'annonce ou d'attente). Une telle structure transforme de fond en comble le statut fonctionnel de cette zone intermédiaire, qui cesse d'être une zone neutre pour participer à la fois de l'effet de surprise, qu'elle doit prolonger, et de l'effet d'annonce, qu'elle doit préparer.

Enfin, Hergé était passé maître dans l'art du récit «étagé », capable de répondre aux désirs des publics les plus variés (le groupe des lecteurs de « 7 à 77 ans » n'était pas fait que d'enfants et d'enfants attardés) comme de tenir compte des sollicitations et des contraintes des deux grands formats de publication, à savoir la prépublication par planches en magazine et la publication intégrale en album. Si les meilleurs livres d'Hergé se prêtent aussi bien à cette lecture en feuilleton qu'à celle en album, c'est justement à cause de la polyvalence des planches mêmes, dont l'effet narratif ne dépend jamais seulement de la seule logique de l'attente et de la surprise hebdomadaires. Comme la logique du feuilleton est atténuée, pour se disséminer sur tous les strips, voire sur toutes les cases (y compris les cases «intermédiaires»), les Aventures de Tintin absorbent sans difficulté la perte en termes de tension narrative que suppose l'intégration des livraisons en une structure d'ensemble, tout en gardant suffisamment d'effets microscopiques, à l'échelle du strip, pour faire de la lecture de l'album - et surtout de la relecture, quand bien même elle commencerait à n'importe quelle page du livre - une entreprise durablement passionnante.

À l'instar de ce qui a été analysé à hauteur des structures énonciatives de l'autobiographie, la bande dessinée littéraire ou le roman graphique se révèlent donc à même de relancer le débat sur notre vision de l'esthétique du feuilleton. Comme le montre un auteur comme Hergé - représentatif en cela du média dont il a inventé bien des règles -, le feuilleton n'est ni une forme littéraire pauvre, ni une pratique obsolète. En ce sens, les Aventures de Tintin peuvent non seulement donner bien des idées à ceux et celles qui ont envie de se tourner à nouveau vers le feuilleton, mais aussi et surtout inviter les théoriciens de la littérature à rouvrir le dossier de ce genre injustement condamné aux marges de la paralittérature.

\section{NOTES}

1. Yves-Marie Labé, « Les bulles et les lettres. Les adaptations dessinées d'œuvres littéraires se multiplient. », in Le Monde des Livres, Vendredi 28 mars 2008. 
2. Comme il s'agit à mes yeux d'un débat un rien différent, je préfère ici laisser de côté la discussion sur les frontières, peut-être inexistantes, entre bande dessinée et roman graphique (ce qui ne veut évidemment pas dire que cette discussion ne tire pas à conséquence : la percée du label « roman graphique » en dit long sur le tournant littéraire de la bande dessinée en général). Pour cette raison, le présent article ne fera pas de distinction terminologique entre « roman graphique » et « bande dessinée littéraire ». 3. Le projet, à paraître en 2009 aux PU de Cambridge, New York, est placé sous la direction de Leonard Cassuto, Clare Eby et Benjamin Reiss.

4. Pour une synthèse récente, voir Jared Gardner, "From the Editor's Chair”, in American Periodicals, vol. 17 n. 2, 2007, pp. 139-141.

5. Pour un exemple récent, voir le numéro spécial de MEI, « Poétiques de la bande dessinée », No 26, 2007, dirigé par Pierre Fresnault-Deruelle et Jacques Samson.

6. Cf. Deborah Cartmell et Imelda Whelehan, dir., Adaptation, Londres, Routledge, 1999, et Linda Hutcheon, A Theory of Adaptation, New York, Routledge, 2007.

7. Un exemple presque caricatural est représenté par le travail du dessinateur hollandais Dick Matena, qui s'est spécialisé dans la transposition en forme de bande dessinée de textes littéraires complets, dont le texte intégral est repris à l'intérieur des cases. J'ai discuté ce sous-genre un peu extrême dans mon article "La bande dessinée 'littéraire' : une nouvelle chance pour la littérature, un danger pour la bande dessinée ?", Contemporary French Civilization 28-2, 2004, pp. 253-273.

8. Raymond Williams, Televsion. Technology and Cultural Form, Londres, BFI, 1990 (1ère édition 1974).

9. Je pense par exemple Thomas Elsaesser et à son analyse foucaldienne du cinéma dans son article Thomas Elsaesser, "Early Film and Multi-media", in Wendy Hui Kuyong \& Thomas Keenan, New Media, Old Media, Londres, Routledge, 2006, pp. 13-26

10. Pour une discussion très décapante de ces questions, voir Noel Carroll, Theorizing the moving image, New York, Cambridge University Press, 1996 (voir surtout le 1er chapitre, "Medium specificity arguments and the self-consciously invented arts: film, video, and photography", pp. 3-24). Pour un exemple dans le domaine de la bande dessinée, voir mon analyse de la bande dessinée flamande dite de l'âge d'or (1945-1965), dont j'ai essayé de démontrer qu'à la lumière du contexte politique et éditorial il est plus logique de l'interpréter comme une variante du média « cartoon politique » que comme une réinterprétation locale de la Ligne Claire établie par l'École de Bruxelles : «Vue de Belgique francophone, la bande dessinée flamande n'est pas une... bande dessinée ", in Textyles, 30, 2007, pp. 117-121

11. On peut penser par exemple à l'adaptation du Château de Kafka dans le roman graphique (plus précisément : gravé sur bois) éponyme de l'artiste belge Olivier Deprez, publié en 2002 aux éditions FRMK à Bruxelles.

12. C'est, on le sait, le cas de certaines bandes dessinées d'avant-garde, dont en tout premier lieu La Cage de Martin-Vaughn, paru d'abord en anglais en 1975, mais devenu célèbre grâce à sa traduction française (Bruxelles, Les Impressions Nouvelles, 1984, puis, dans une édition légèrement différente, 2005).

13. Sur toutes ces questions, la meilleure introduction reste le livre de Benoit Peeters, Case, planche, récit, Paris, Casterman, 1998.

14. Bien entendu, la bande dessinée muette est tout sauf l'apanage de la seule avantgarde, comme l'a bien montré Thierry Groensteen dans ses deux études, « Histoire de la bande dessinée muette I - II », $9 e$ Art, No 2, 1997, pp. 60-75, et No 3, 1998, pp. 92-105, ou comme l'indique aussi l'ouvrage historique de David Beronå, Wordless Books: The Original 
Graphic Novels, New York, Harry N. Abrams, 2008. Cependant, l'avant-garde semble avoir une prédilection certaine pour les livres «sans paroles».

15. Le cas déjà cité de La Cage serait ici exemplaire.

16. Pour une discussion de cette question, (re)lancée par l'ouvrage de Spiegelman à

l'ombre des tours mortes, voir Charles Hatfield, Alternative Comics. An Emerging Literature, Jackson: Mississipi University Press, 2005, p. 162, et Hillary Chute, "Temporality and Seriality in Spiegelman's In the Shadow of No Towers", in American Periodicals 17-2, 2007, pp. 228-244.

17. Pour une discussion de cette question, voir mon article: « Une photographie vaut-elle mille films ? », in « Actualités du récit. Pratiques, théories, modèles », Protée vol. 34-2/3, 2006, pp. 67-76, où je compare un film de Cartier-Bresson et une image tirée de la première oeuvre, qui montre bien, je crois, à quel point l'image fixe peut s'avérer plus narrative que l'image mobile.

18. Voir son article "Narratologie médiatique et médiagénie des récits", in Recherches en communication, No 7, 1997, pp. 61-87.

19. Voir son essai Traces en cases, Louvain-la-Neuve, Académia, 1993

20. Trois volumes, Paris, L'association, respectivement 2000, 2002 et 2008. À signaler que cet auteur est familier des expériences en médiagénie puisqu'il a également publié une série d'albums mélangeant dessins et photographies : Le photographe, en collaboration avec Didier Lefèvre, Paris, Dupuis, 2003-2005 (3 tomes).

21. Voir Raphaël Baroni, La tension narrative. Suspense, curiosité, surprise, Paris, Seuil, 2007. 22. C'est, dans un autre media et un tout autre genre, le problème du très grand scénariste qu'est David Mamet dans son film Heist: à force de miser sur les retournements de situation trop surprenants, le spectateur finit par s'en désintéresser.

\section{RÉSUMÉS}

Le présent article se propose d'examiner deux aspects majeurs de la rencontre entre littérature et bande dessinée, dont le champ ne peut être limité à celui des seules adaptations de textes littéraires en bande dessinée. D'une part, il propose une définition de la bande dessinée littéraire (ou du roman graphique, peu importe la terminologie) qui met l'accent sur le concept de récit, plus particulièrement sur le concept de récit visuel, pour analyser ensuite quels types de récits visuels sont considérés comme littéraires en bande dessinée. D'autre part, il démontre que l'analyse littéraire de la bande dessinée peut aider considérablement à renouveler l'étude de certains aspects de la théorie narrative, notamment dans le domaine du discours autobiographique et des techniques du feuilleton.

This article deals with two crucial aspects of the encounter between literature and comics, which cannot be reduced to the mere problem of the adaptation of literary texts in comics. On the one hand, it offers a new definition of the literary comics (or graphic novel, although this kind of terminological discussions will be avoided here), which puts a strong emphasis on the concept of storytelling, more precisely of visual storytelling, and it analyzes which types of storytelling may be considered literary in the comics field. On the other hand, it argues that the literary reading of comics may prove dramatically helpful in the elaboration of new ways of interpreting 
narrative theory, for instance in the domain of autobiography as well as in the domain of the instalment techniques.

INDEX

Mots-clés : autobiographie, bande dessinée, feuilleton, polyphonie, roman graphique Keywords : graphic novel

Index chronologique : XXe siècle

\section{AUTEUR}

JAN BAETENS

Université de Leuven, Institut d'études culturelles 